\title{
LA RIBELLIONE COME ATtO CREATIVO. IL POTERE DEL DIRITTO E DELLE STRUTTURE NELLA TEORIA DEI SISTEMI DI NIKLAS LUHMANN
}

\author{
Corrado Punzi ${ }^{1}$ \\ Università del Salento, Italia
}

\begin{abstract}
Definendo l'uomo come ambiente del sistema sociale, Luhmann ha determinato una rivoluzione epistemologica che ha provocato e continua a provocare ampie critiche da parte del pensiero sociologico tradizionale. Attuando un'analisi priva di pregiudizi umanistici, è possibile, però, vedere nella teoria dei sistemi di Luhmann una sconvolgente modernità. Ed è possibile vedere come il controllo esercitato dal diritto non sia altro che un controllo operante in ogni sistema sociale, in ogni tipo di relazione umana, tant'è che the control's control is the system. Per ribellarsi, quindi, non è sufficiente opporsi al potere o al diritto, ma pensare nuove strutture: rendersi creativi o diabolici, rischiando di essere espulsi da questo mai raggiunto paradiso terrestre che non prevede altri creatori.
\end{abstract}

By defining man as environment (of the social system), Luhmann produced an epistemological revolution that has caused and continues to provoke widespread criticism from the traditional sociological thought. Carrying out an analysis without prejudice, it is possible, however, to see a shocking modernity in Luhmann's systems theory. And it is possible to see that the control exercised by the law is just playing a control that takes place in any social system, in every kind of human relationship, such that the control's control is the system. And so, to rebel is not sufficient to fight the power or the law, but to think of new structures: to become creatives or devils, assuming the risk of being expelled from this (never reached) paradise on earth that does not include other creators.

Keywords.- Luhmann, systems theory, control, law, structure, rebellion, creative act

L'innovativa concezione del potere e del diritto di Niklas Luhmann è conseguente alla sua rivoluzionaria concezione della società in chiave sistemica. Una rivoluzione epistemologica di tale rilievo che ha indotto a considerare la sua teoria della società «una concettuologia delle scienze sociali», poiché ridefinisce, e quindi ri-problematizza, tutti i concetti cardine della sociologia e del pensiero giuridico-politico della vecchia Europa: l'idea di società, di azione, di razionalità, etc. Una rivoluzione di così ampia portata da generare inevitabilmente ampie critiche, ma anche l'ammissione, altrettanto

$1 \quad$ Corrado Punzi è dottore di ricerca in Scienze giuridiche. Si occupa di diritto e opinione pubblica e analizza il potere come medium della comunicazione. Ha svolto periodi di ricerca in Cile, Brasile, Burundi e Spagna, analizzando i processi di democratizzazione nelle periferie della modernità e il tema dei diritti umani. Attualmente svolge attività di ricerca presso il Centro di studi sul rischio di Lecce fondato da Raffaele De Giorgi e Niklas Luhmann. Ha scritto un libro dal titolo Democrazia come paradosso. Sentieri convergenti: Luhmann, Foucault, Canetti, Pensa, Lecce 2011 [in corso di pubblicazione] 
inevitabile, di trovarsi di fronte ad uno dei massimi pensatori del Novecento, la cui produzione sterminata (46 opere monografiche tradotte in 15 lingue e 417 articoli scientifici), non può essere interpretata solo in chiave sociologica 0 giuridica, ma anche filosofica. ${ }^{2}$

L'idea principale alla base della rivoluzione luhmanniana è che la società non è costituita da uomini: I'uomo è ridotto a sistema psichico (e corpo organico) e, in quanto tale, è ambiente del sistema sociale, così come il sistema sociale appartiene all'ambiente dei sistemi psichici. Gli uomini, in quanto sistemi psichici, vengono quindi lasciati al monopolio della psicologia a discapito della sociologia; alla biologia, invece, resta l'analisi degli altri sistemi esistenti: quelli organici. Quindi: sistemi organici, sistemi sociali ${ }^{3}$ e sistemi psichici.

«E' chiaro che non tutto ciò che si può osservare nell'uomo (ammesso che qualcosa si possa osservare) appartiene alla società. La società non pesa quanto tutti gli uomini messi insieme e non cambia il suo peso per ogni uomo che nasca o per ogni uomo che muoia. Essa non si riproduce per il fatto che nelle singole cellule dell'uomo vengano scambiate macromolecole o per il fatto che negli organismi dei singoli uomini vengano scambiate cellule. La società non vive. Né si vorranno intendere sul serio come processi sociali i processi neurofisiologici del cervello, che sono inaccessibili alla coscienza stessa; lo stesso può dirsi di tutto ciò che si verifica nell'ambito attuale di attenzione della singola coscienza, siano esse percezioni o successioni di pensieri. II fatto che, nonostante tutte queste evidenze, si resti fermi ad un concetto "umanistico" di società, cioè ad un concetto che ha nell'uomo il suo riferimento essenziale, è probabilmente condizionato dal timore che, diversamente, ci si sentirebbe privi di ogni misura di valutazione della società e quindi non si avrebbe più diritto a pretendere che la società debba essere organizzata "in modo umano". Anche se così fosse, sarebbe pur sempre necessario, indipendentemente da tali criteri, poter stabilire prima di tutto che cosa la società fa degli uomini e perché questo accade» ${ }^{4}$.

L'idea che la società non vive e non è costituita da uomini priva la sociologia del suo stesso oggetto e per questo non può non provocare ampie critiche. Se tuttavia si riescono ad arginare i pregiudizi e ci si sforza di entrare nella cosiddetta "astrattezza formale" della costruzione teorica luhmanniana, si comprende parte dell'attualità e del fascino di un pensiero che non dimentica l'uomo, ma rinuncia a pensare la società moderna a partire dall'uomo. Il fascino e l'attualità di tale pensiero derivano, a nostro avviso, dal fatto che questa rinuncia all'uomo è la conseguenza logica di una teoria che non separa soggetto e oggetto della conoscenza, perché reputa irrisolvibile, o meglio mal

2 Lo stesso sociologo del diritto Alberto Febbrajo, dopo aver espresso nel corso degli anni numerose critiche nei confronti della teoria sistemica della società, ha riconosciuto che Luhmann è un «giurista-sociologo-filosofo» e che le critiche nei suoi confronti «semplicemente scambiano per difetti e carenze ciò che, nell'ottica di Luhmann, è invece il risultato di una consapevole scelta di diversificazione operata non solo nei contenuti ma anche delle pretese esplicative di altre teorie». Cfr. A. Febbrajo, Introduzione all'edizione italiana, in N. Luhmann, Sistemi sociali. Fondamenti di una teoria generale, II Mulino, Bologna 1990, p. 9 e p. 38

Luhmann distingue tre tipi di sistema sociale: l'interazione; l'organizzazione; la società. Per questo, mentre - come vedremo -, in Parsons si parla al singolare di "sistema sociale", in Luhmann si utilizza il plurale.

N. Luhmann - R. De Giorgi, Teoria della società, Franco Angeli, Milano 2003, pp. 14-15 
posto, il problema weberiano dell'avalutatività scientifica. ${ }^{5}$ Secondo Luhmann, infatti, la sociologia classica, da Weber a Parsons, ha sempre presupposto, come criterio di scientificità, l'assenza di vincoli tra sociologia e società e quindi ha sempre lasciato

«aperte le questioni dell'autoimplicazione cognitiva [...]. Essa postula soltanto un "realismo analitico" e condensa così in una formula paradossale il problema dell'autoimplicazione. Essa non tiene presente che la conoscenza dei sistemi sociali dipende da condizioni sociali non soltanto per il suo oggetto, ma già come conoscenza» ${ }^{6}$.

Rifacendosi all'analisi logica della linguistica, Luhmann ritiene che ogni teoria della società debba presentare una componente "autologica", poiché già la definizione di cosa sia società è una delle operazioni della società. La sociologia, insomma, non può osservare la società dall'esterno, essendo essa stessa società. La società, infatti, intesa «come sistema sociale complessivo, non conosce sistemi sociali oltre i suoi confini»» ${ }^{7}$. Per questa ragione, ogni descrizione sociale deve descrivere anche se stessa, realizzando un rapporto circolare con il suo oggetto, tramite l'autoinclusione.

Da questa prospettiva, è plausibile ritenere, pertanto, che il rifiuto luhmanniano di ogni concezione umanistica sia complementare alla descrizione della modernità: non sarebbe quindi la teoria dei sistemi ad aver "espulso" l'uomo, ma sarebbe stata la società stessa a farlo. Luhmann, infatti, afferma che in «società più antiche può ipotizzarsi una differenziazione più limitata tra strutture sociali e strutture psichiche. Tali società devono poggiare il loro patrimonio normativo [...] su strutture psichiche relativamente concrete [...]. La società moderna, per contro, ha così accentuatamente individualizzato le strutture della personalità $[. .$.$] che è diventato necessario istituire una distinzione più netta e$ una reciproca indifferenza tra strutture psichiche e sociali» ${ }^{8}$. Parte del fascino e dell'attualità della teoria di Luhmann, quindi, deriva dal fatto che essa non si limita a descrivere la modernità della società moderna, a riscontrare - come aveva fatto Durkheim - le sue conseguenze sulla divisione del lavoro o - come Weber - sull'uomo rinchiuso nella gabbia d'acciaio della burocrazia. La teoria dei sistemi va oltre la semplice osservazione della modernità, perché realizza un rapporto circolare con il suo oggetto, creando un'identificazione tra problema e teoria. Pertanto, si può azzardatamente ritenere, ad esempio, che il problema della divisione del lavoro o quello della burocratizzazione della vita, vengano non solo riformulati concettualmente all'interno di una prospettiva completamente differente, ma anche applicati alla stessa teoria, che infatti descrive la società moderna come sistema sociale differenziato per funzioni e, come conseguenza delle sue osservazioni, relega l'uomo in una sorta di metaforica gabbia, che definisce ambiente. Ecco «che cosa la società fa degli

$5 \quad$ Secondo Luhmann anche l'avalutatività è un valore e quindi «Tutto ciò che si può fare è garantire l'indipendenza della teoria da valutazioni che appartengono ad altri sistemi funzionali, di tipo religioso, estetico, politico, economico, pedagogico, ecc. II modo migliore di far ciò non consiste nel negare l'esistenza di connessioni di valore [...], ma nella precisazione dei propri riferimenti di valore». N. Luhmann, Illuminismo sociologico, II Saggiatore, Milano 1983, p. XL

Ivi, p. 12

Ivi, p. 29

N. Luhmann, Sociologia del diritto, Laterza, Roma-Bari 1977, p. 262 
uomini», ma anche che cosa la società fa di una teoria sociale che presenti una componente autologica.

Si può ritenere, pertanto, che la modernità della teoria dei sistemi consista nell'applicare il processo di de-soggettivazione osservato nella società moderna alla stessa teoria: così, anche il concetto di soggetto viene sostituito con quello di sistema autoreferenziale, «che, non essendo necessariamente un sistema psichico, non esegue necessariamente le proprie operazioni sotto forma di coscienza» $^{9}$. D'altra parte, tale nuovo concetto «si addice meglio alla visione acentrica del mondo che è propria della scienza odierna» ${ }^{10}$. L'operazione di autoriferimento «risulta inclusa in ciò che designa. Essa designa qualcosa del quale essa stessa fa parte. [...] Essa designa, sempre sotto la guida di una distinzione, qualcosa con cui essa si identifica» ${ }^{11}$. La teoria dei sistemi, pertanto, si identifica con ciò che essa osserva: la sua modernità consiste - a nostro avviso -, nella capacità di guardare oltre l'uomo e di trasformare la rivoluzione, prima burocratica e poi informatica, in un quadro teorico che sostituisce alla tradizionale metafora dell'organismo o della macchina un'immagine della società più vicina al computer. Come spiega infatti Febbrajo,

«Ciò in effetti non fa che rafforzare l'orientamento anti-soggettivistico [...] in quanto la società-computer e la società-robot, a differenza della societàmacchina e della società-organismo, ha la caratteristica di trattare problemi tradizionali della sociologia senza ricorrere alla ipotesi di un qualche soggetto individuale o collettivo con funzioni ordinatrici, essendo essa stessa capace, per definizione, di svolgere tali funzioni, e quindi di essere ad un tempo oggetto e soggetto» ${ }^{12}$.

Nella sua visione acentrica, Luhmann non cerca «un qualche soggetto individuale o collettivo con funzioni ordinatrici»: eliminando il soggetto, il problema dell'ordine - e quindi del potere -, non può più essere posto secondo la vecchia semantica hobbesiana, non può più essere risolto con una vana ricerca di un soggetto o di un luogo di sovranità. «ll vecchio problema di evitare l'arbitrio (despotie) del vertice [...] passa in seconda linea. Chi controlla il controllore, ci si chiedeva inutilmente. Oggi la risposta è: The control's control is the system».13

\footnotetext{
9

10 N. Luhmann, Sistemi sociali. Fondamenti di una teoria generale, cit., p. 673
}

$10 \quad$ Ibidem

$11 \quad$ Ivi, p. 677

12 A. Febbrajo, Introduzione all'edizione italiana, in N. Luhmann, Sistemi sociali. Fondamenti di una teoria generale, cit., p. 15

13 N. Luhmann, Teoria politica nello Stato del benessere, Franco Angeli, 1987, p. 80. La teoria dei sistemi subisce il fascino non solo della biologia o dell'antropologia funzionalista, ma anche della cibernetica. L'espressione The control's control is the system è di Glanville: è lui a rivalorizzare la fenomenologia dell'osservazione e a suggerire di adottare una cibernetica di secondo ordine (osservazione dell'osservazione; controllo del controllo), perché in questo modo è possibile includere l'osservatore nel sistema, autoimplicarlo e così superare definitivamente il problema dell'avalutatività. Come spiega Sutter, «l'osservatore non descrive solo il sistema [...], ma descrive e trasforma input ed output. L'osservatore opera come controllo cibernetico e come sistema di comunicazione per il sistema osservato e non lo si può più considerare come sistema indipendente e isolato. La formulazione essenziale della cibernetica di secondo grado è quindi l'unità di osservato e osservatore. Un modo di concepire questo modello è l'idea di autoreferenza: il sistema è costituito da se stesso osservante se stesso. E l'osservazione fatta dal sé (autosservazione) è diversa dall'osservazione fatta dagli altri; attraverso l'autoreferenza il 
II problema, a questo punto, è comprendere che cosa Luhmann intenda con sistema, che è l'altra parte di ambiente. La distinzione sistema/ambiente diventa, quindi, innanzitutto fondamentale per comprendere la relazione tra uomini e sistema sociale, poiché essa sostituisce la distinzione parte/tutto, tramite la quale la sociologia classica pensava la società come costituita dalla somma di tutti gli uomini che ne fanno parte. Tuttavia, se gli uomini sono descritti come ambiente del sistema sociale, è rilevante chiedersi da cosa sia costituita la società e quindi quale sia l'oggetto d'analisi della sociologia luhmanniana. In definitiva, da cosa sia costituito quel sistema sociale chiamato società.

Innanzitutto, è bene precisare che la teoria dei sistemi di Luhmann, rinunciando al concetto di soggetto, rinuncia anche all'idea di oggetto: «non parliamo più di oggetti ma di distinzioni» ${ }^{14}$. In questo modo è possibile evitare qualsiasi presupposto sostanzialistico su cui si fondava la sociologia classica. La sostituzione del concetto di oggetto con quello di distinzione rimanda all'affermazione di Goethe, secondo cui «Ogni parola che si pronuncia fa pensare al suo contrario». ${ }^{15}$ II concetto di distinzione è direttamente correlato a quello di forma:

«La forma è forma di una distinzione, quindi di una separazione, di una differenza. Si opera una distinzione tracciando una demarcazione che separa due parti, per cui non si può passare da una parte all'altra senza attraversare la demarcazione. Forma è allora una linea di confine che segna una differenza e costringe a chiarire quale parte si indica quando si dice che ci si trova da una parte [...]. Quando si effettua una distinzione, si indica una parte della forma; con essa però è data allo stesso tempo l'altra parte: è data cioè contemporaneità e differenza temporale. Indicare è insieme: distinguere, così come: distinguere è insieme: indicare. Ogni parte della forma, allora, è l'altra parte dell'altra parte. Nessuna parte è qualcosa per se stessa» ${ }^{16}$.

Sulla base di questa idea di forma come forma di una distinzione, Luhmann utilizza la distinzione sistema/ambiente per costruire la sua teoria e per operare una molteplicità di distinzioni: ogni sistema, infatti, se è sufficientemente complesso, può applicare a se stesso la distinzione sistema/ambiente. Cosa si indica, allora, quando si indica qualcosa con sistema?

Già prima di Luhmann, Parsons aveva descritto la società come sistema sociale e lo stesso Luhmann, ad inizio anni sessanta, si era recato all'Università di Harvard per studiare proprio con Parsons, allora direttore del Dipartimento di Relazioni Sociali. Tale scelta fu probabilmente dettata dal ritenere la teoria struttural-funzionalista parsonsiana, il contributo più fecondo all'adozione di una nuova epistemologia, poiché, per prima, situa l'azione nell'ottica del sistema. ${ }^{17}$ Struttura, funzione, sistema: concetti che già affascinano Luhmann, ma che egli è destinato a ri-problematizzare, modificandone la semantica. Come afferma lo stesso Luhmann, la teoria struttural-funzionalista di Parsons

sistema genera i propri confini e mantiene l'autonomia individuale». R. Sutter, Introduzione, in N. Luhmann, Teoria politica nello Stato del benessere, cit., p. 32

$14 \quad$ N. Luhmann - R. De Giorgi, Teoria della società, Franco Angeli, Milano 2008, p. 16

$15 \quad$ Ivi, p. 73; Cfr. Goethe, Le affinità elettive, Torino 1966, p. 187

$16 \quad$ IVi, p. 17

17 Cfr. N. Luhmann, Come è possibile l'ordine sociale, Laterza, Roma-Bari 1985, p. 95 
«comportava troppe scelte preliminari di carattere problematico. Penso, ad esempio, all'analisi del concetto di azione scelta da Parsons come punto di partenza della sua analisi [...]. Penso alla tecnica di tabulazione incrociata ed alla rigidità della suddivisione dell'esperienza sociale in quattro aree distinte. In una tale situazione mi sembrava indispensabile un ripensamento che avesse una portata insieme teorica e metodologica» ${ }^{18}$.

La revisione luhmanniana della teoria struttural-funzionalista, d'altra parte, è anche un tentativo di costruire una teoria che possa essere immune all'accusa di conservatorismo ricevuta da Parsons. Luhmann, infatti, ritiene che Parsons si sia esposto alle critiche perché si è concentrato sul contributo che i singoli sottosistemi forniscono in vista della conservazione delle strutture del sistema sociale. II problema centrale di Parsons, cioè, è la stabilità del sistema sociale ottenibile tramite l'individuazione di specifiche funzioni che garantiscano la permanenza delle strutture esistenti. Concentrandosi su questo aspetto, il sociologo statunitense si è facilmente esposto all'accusa di essere solo interessato alla conservazione delle strutture e quindi dello status quo.

«Per levare fondamento alle motivazioni di tale accusa, Luhmann propone di invertire l'ordine logico dei concetti parsonsiani e di premettere il concetto di funzione a quello di struttura. Egli, pertanto, non considera più le strutture sociali come dati ultimativi, ma si chiede, da un punto di vista funzionale, quale sia il loro senso in determinate situazioni. II problema fondamentale della ricerca sociologica, insomma, non è più, come per Parsons, la determinazione delle condizioni da realizzare perché certe strutture continuino a sussistere, ma, al contrario, la determinazione della funzione che tali strutture svolgono in un mondo difficile. II punto di partenza dell'approccio struttural-funzionalistico parsonsiano risulterebbe, così, capovolto» ${ }^{19}$.

Questo capovolgimento segna il passaggio dallo struttural-funzionalismo al funzional-strutturalismo.

Luhmann contesta a Parsons il fatto che la sua costruzione teorica derivi le funzioni dalle strutture, considerando quindi la struttura come elemento statico del sistema e la funzione come elemento dinamico. Secondo Luhmann, invece, le strutture non possono essere presupposte, ma è necessario che anche esse siano problematizzate e consegnate alla possibilità del mutamento. ${ }^{20}$ II concetto parsonsiano di funzione, al contrario, è «strettamente connesso ad uno schema causale unilineare, poiché, data una struttura ed identificata la sua funzione, è possibile dedurre causalmente le attività necessarie al soddisfacimento dei bisogni del sistemas» ${ }^{21}$. Secondo Luhmann, invece, la funzione non può essere ritenuta, come fanno i funzionalisti, un effetto che deve essere prodotto da una struttura già pre-definita, perché «la funzione non è un tipo particolare di relazione causale ${ }^{22}$. E' per questo che arriva a sostenere sia necessario non adottare una concezione biologica di funzione, ma logico-matematica, in modo che anche le scienze sociali possano acquisire i nuovi orizzonti di senso aperti

\footnotetext{
18 N. Luhmann, Illuminismo sociologico, II Saggiatore, Milano 1983, p. XXXV

19 A. Febbrajo, Funzionalismo strutturale e sociologia del diritto nell'opera di Niklas Luhmann, Giuffrè Editore, Milano 1975, p. 30

$20 \quad$ Cfr. N. Luhmann, Illuminismo sociologico, cit., p. 34

21 G. Palombella-L. Pannarale, Introduzione all'edizione italiana in N. Luhmann [1999], I diritti fondamentali come istituzione, Edizioni Dedalo, Bari 2002, pp. 9-10

22 N. Luhmann, Illuminismo sociologico, cit., p. 16
} 
dalla fisica quantistica e cioè l'idea di una complessità che non può essere indagata tramite una logica causale e unilineare. Adottando il concetto matematico di funzione, Luhmann abbandona il funzionalismo causalistico e lo sostituisce con il funzionalismo delle equivalenze. ${ }^{23}$ In questo modo, l'osservazione sociologica non si concentra più soltanto «su due fenomeni, la causa e l'effetto, ma su un fascio di fenomeni la cui caratteristica comune è quella di poter produrre, indipendentemente l'uno dall'altro, un medesimo effetto» ${ }^{24}$. La sociologia, quindi, ha il compito di comprendere com'è possibile che tra tutte le funzioni equivalenti se ne affermi una, ma anche comprendere cosa accade a tutte le altre possibilità che sono rimaste latenti. Luhmann, quindi, mutua dalla psicanalisi il concetto di latenza, ma non allo scopo di comprendere le ragioni inconsce dell'agire, quanto per capire la funzione che la latenza assume in un sistema sociale e se si possano individuare delle alternative funzionalmente equivalenti. ${ }^{25}$

Nonostante il funzionalismo di Luhmann si occupi, come quello parsonsiano, del problema della stabilità del sistema, spezzando il determinismo unilineare tra struttura e funzione, rifiuta la considerazione ontologica della stabilità. ${ }^{26}$ Ciò vuol dire che mentre Parsons pensa ci sia prima la struttura e poi le operazioni che la stabilizzano, Luhmann invece ritiene ci siano delle operazioni che si differenziano e si stabilizzano come struttura di un sistema. Adottando il concetto di autopoiesi elaborato dal biologo Humberto Maturana, Luhmann descrive i sistemi come sistemi autopoietici, cioè «sistemi che producono essi stessi non soltanto le loro strutture, ma anche gli elementi dei quali essi sono composti». ${ }^{27}$ All'unilinearità subentra la circolarità: un elemento condiziona un altro elemento, ma ne è, a sua volta, condizionato. La struttura è, pertanto, il risultato delle operazioni che essa connette e che la rendono possibile. L'idea luhmanniana di sistema è l'idea di una circolarità che si produce da sé. Quali sono, allora, le operazioni di una struttura? Qual è, quindi, l'oggetto d'analisi della sociologia luhmanniana?

Le operazioni o gli elementi di un sistema sociale sono comunicazioni. La società è quindi per Luhmann il sistema sociale complessivo che si identifica con l'universo della comunicazione sociale e, come gli altri sistemi (viventi e psichici), non può essere data indipendentemente dal suo ambiente. Sulla base di questa idea, la teoria della società di Luhmann può essere definita una teoria della comunicazione: innovativa perché - come si è detto -, descrive la società non come l'insieme degli uomini, ma sulla base delle loro comunicazioni, analizzate in quanto tali, cioè indipendenti dalle motivazioni che le producono. «Quando parliamo di ambiente ci riferiamo a tutto ciò che non è nel contesto di operazioni che si isolano e si chiudono come universo della comunicazione sociale; quindi dicendo ambiente, intendiamo uomini in carne ed ossa, intendiamo cervelli, intendiamo corpi»s. ${ }^{28}$ II funzionalismo di Luhmann, quindi, si

23 Il funzionalismo delle equivalenze non è, però, un'innovazione concettuale di Luhmann: Merton, infatti, utilizza già prima il concetto di equivalente funzionale.

${ }_{24}$ A. Febbrajo, Funzionalismo strutturale e sociologia del diritto nell'opera di Niklas Luhmann, cit., p. 32

${ }_{25} \quad$ Cfr. N. Luhmann, Illuminismo sociologico, cit., pp. 77-78

26 R. De Giorgi, Scienza del diritto e legittimazione sociale, Pensa Multimedia, Lecce 
differenzia da quello di Parsons soprattutto perché il sociologo statunitense continua ad adottare una prospettiva individualistica, ancorata alle idee weberiane di azione, di razionalità individuale e di agire dotato di senso. Luhmann, tuttavia, proponendo una visione antiumanistica e, contemporaneamente, antiregionalistica della società (la società è l'universo della comunicazione sociale), non nega che esistano uomini e non ignora neppure le forti distinzioni che segnano le condizioni della vita in diversi luoghi del globo terrestre. Egli rinuncia «soltanto a derivare da questi fatti un criterio per la definizione del concetto di società e per la determinazione dei confini dell'oggetto che ad esso corrisponde» ${ }^{29}$. Gli uomini, in quanto sistemi psichici, non sono presi direttamente in considerazione perché essi anche quando pensano la società e la osservano, lo fanno in quanto ambiente dei sistemi sociali e quindi dall'esterno rispetto ad essi; ciò che avviene nei sistemi psichici, socialmente, «resta senza conseguenze se non si comunica, se cioè l'osservazione non viene praticata nel sistema sociale». ${ }^{30} \mathrm{E}$ ' chiaro, quindi, che le comunicazioni, in quanto elementi dei sistemi sociali, presuppongono il concorso di un gran numero di sistemi di coscienza; tuttavia, la comunicazione «è sociale perché in nessun modo può essere prodotta una coscienza "comune" collettiva, cioè non può essere raggiunto il consenso nel senso di un accordo completo: eppure la comunicazione funzionas ${ }^{31}$.

Scegliendo come oggetto di analisi le comunicazioni e non gli uomini o le loro motivazioni, Luhmann adotta quella che Kenneth Burke ha definito perspective by incongruity, perché solo tramite una prospettiva incongrua e discrepante il «senso dell'azione cessa di essere spiegato attraverso l'approfondimento della sua essenza, del suo telos» ${ }^{32}$. Luhmann, quindi, rifiuta il compito - assunto dalla filosofia pratica -, di spiegare all'individuo agente i suoi scopi veri e di indicare i mezzi per raggiungerli, così come rifiuta il riduzionismo soggettivistico di Weber o quello oggettivistico di Durkheim. Egli, infatti, vuole liberare la scienza dalla funzione di ricercare un'unità del senso e intende trovare per le scienze sociali una via analoga a quella che hanno perseguito alcune correnti artistiche del novecento tramite le tecniche estranianti. A suo avviso, quindi, soltanto tramite una prospettiva discrepante, «il senso stesso perde il suo carattere compatto, impenetrabile, sostanziale, la sua capacità di fondare verità ${ }^{33}$. II concetto di senso non può più essere dato per scontato, cioè non può più essere considerato come «una qualità (se pure soggettiva) delle azioni» ${ }^{34}$. Solo così, il senso, come il mondo, può rivelare la sua contingenza, cioè il suo manifestarsi in un modo, ma poter essere anche in un altro. "Da questo momento è possibile fondare una teoria che abbia un ampio respiro unicamente come proposta di soluzione di questo problema»: la contingenza sociale del mondo. Anche le teorie di Durkheim e Weber, secondo Luhmann, hanno affrontato questo problema, «pur senza nominarlo», ma - come si è detto -, «la loro inadeguatezza sta nel fatto che questo loro problema non si identifica con la loro teoria» ${ }^{35}$. Il problema, quindi, è costruire una teoria che analizzi la

\footnotetext{
29 N. Luhmann, R. De Giorgi, Teoria della società, cit., p. 15

$30 \quad$ Ivi, p. 29

$31 \quad$ Ivi, p. 26

32 N. Luhmann, Illuminismo sociologico, II Saggiatore, Milano 1983, p. 76

$33 \quad$ Ivi, p. 133

$34 \quad$ Ibidem

$35 \quad$ Ivi, p. 76.
} 
contingenza e che diventi essa stessa contingente: una teoria priva di presupposti, che consenta di esporre le sue osservazioni ad altre osservazioni, poiché dice quali distinzioni usa per costruire le sue descrizioni e così evita il problema dei fondamenti e sfugge al rischio dell'ontologia ${ }^{36}$. Tale teoria, attraverso una «opera di disincantamento [...] scopre che la determinazione sociale incide in modo molto più ampio di quanto generalmente si supponeva e di quanto, in particolare, è disposto a riconoscere l'individuo stesso che agisce ${ }^{37}$. Per comprendere che cosa Luhmann intenda per determinazione sociale e in che modo essa incida sulle comunicazioni sociali, è necessario indagare più approfonditamente proprio i concetti di struttura e di sistema, perché è tramite questi concetti che Luhmann tenta di risolvere il problema della contingenza e quello ad esso correlato: la complessità del mondo. Con complessità si indica «che vi sono sempre più possibilità di quelle che sono

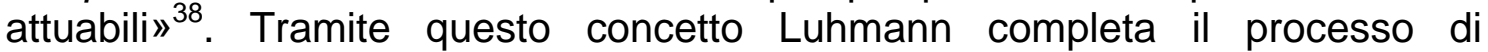
distanziamento della sua teoria da quella di Parsons: il problema di riferimento teorico, infatti, non è più la stabilità. Quest'ultima non è più il problema ultimo, ma soltanto uno dei problemi che precedono il problema ultimo: la complessità del mondo. ${ }^{39}$ La società moderna, pertanto, è complessa perché «offre all'uomo una quantità enorme di possibilità di esperienza e di azione, alla quale egli contrappone soltanto una capacità assai limitata di percepire attualmente e consapevolmente, di elaborare informazioni e di agire» ${ }^{40}$. La contingenza è, quindi, il correlato della complessità, poiché essa evidenzia che, nella totalità delle possibilità, le poche attuabili «possono anche realizzarsi in modo diverso dalle attese ${ }^{41}$. I sistemi sociali, pertanto, hanno lo scopo di presentare una più ridotta complessità rispetto al loro ambiente: «Essi servono a mediare tra la complessità massima del mondo e la capacità molto limitata dell'uomo di assimilare esperienze in modo consapevole» ${ }^{42}$. Questa capacità di mediazione rende ogni sistema medium dell'illuminismo. Un illuminismo radicalmente differente da quello che si afferma nel diciottesimo secolo quando, mentre si esauriva il primato della differenziazione stratificata, si credeva nell'uguale partecipazione di tutti gli uomini ad una comune ragione e si era ottimisti riguardo alla realizzabilità di condizioni sociali giuste. ${ }^{43}$ Queste credenze, secondo Luhmann, si basano su una concezione ingenua dell'illuminismo, che non consente di osservare la società per quello che è, perché nasconde la realtà dietro semantiche che promettono un futuro migliore e, con l'eccessiva luce del loro dover essere, conducono però all'accecamento. Per vedere, o per vedere di non vedere, invece, è necessario adottare un nuovo Illuminismo: quello sociologico. Esso «capovolge il principio ontologico della ragione e instaura il principio funzionale della razionalità ${ }^{44}$. La razionalità che interessa la

\footnotetext{
36 Cfr. R. De Giorgi, Presentazione all'edizione italiana, in N. Luhmann, La fiducia, II Mulino, Bologna 2002, p. XI

$37 \quad$ Ibidem

$38 \quad$ N. Luhmann, Sociologia del diritto, cit., p. 40

39 II vantaggio del funzionalismo delle equivalenze è costituito dalla capacità di sostituire il concetto di stabilità con il concetto di problema, cioè con un criterio di riferimento astratto. Cfr. N. Luhmann, Illuminismo sociologico, cit., p. 37

$40 \quad$ Ibidem

$41 \quad$ Ibidem

$42 \quad$ Ivi, p. 133 [corsivo mio]

$43 \quad$ Ivi, p. 73

$44 \quad$ R. De Giorgi, Scienza del diritto e legittimazione, cit., p. 224
} 
sociologia, non è più quella dell'individuo, ma quella del sistema. Essa così, viene «liberata dalla sua dipendenza causale nei confronti dell'azione» ${ }^{45} \mathrm{e}$ guida il sistema nella risoluzione dei problemi dettati dall'ambiente, consentendo un «ampliamento della capacità umana di cogliere e ridurre la complessità del mondo». ${ }^{46}$ D'altra parte, se una totalità così vasta ed eccedente di possibilità rispetto alle capacità del nostro esperire, non fosse ridotta, renderebbe l'uomo incapace di orientarsi e quindi di agire. La possibilità di orientarsi e di agire deriva quindi dal fatto che i sistemi possono essere definiti anche in modo non formale e, cioè, come sedimentazioni di senso.

Il senso, infatti, secondo Luhmann,

«si produce perché entro un orizzonte di possibilità, attraverso le operazioni di comunicazione, si blocca una di queste possibilità. II fatto che si sia bloccata una delle possibilità, non significa che il senso si sia determinato una volta per tutte, per cui si possa dire con l'attività ermeneutica: questo è il senso. Nell'orizzonte delle possibilità, la determinazione prodottasi rinvia ad infinite altre possibilità. Senso ulteriore si produce, in base ad ulteriori determinazioni che rinviano all'infinito ad altre possibilità. Ne deriva che l'attività di produzione di senso è un'attività di produzione di socialità» 47

Così, il confine tra sistema e ambiente è un semplice confine di senso; un confine che distingue un'identità problematica: il concetto di sistema - chiarisce infatti Luhmann -, va inteso «come un modo ben determinato di risolvere il problema dell'identità, e cioè ricorrendo all'introduzione di una distinzione fra un dentro e un fuori» ${ }^{48}$. L'identità si costituisce sempre come differenza: in questo caso, come differenza di senso tra sistema e ambiente: «al principio non sta l'identità, ma la differenza» ${ }^{49}$. Pertanto, l'identità di un sistema è possibile solo come differenza da un altro sistema: la «formazione di un sistema si attua attraverso la stabilizzazione di un confine tra sistema e ambiente» ${ }^{50}$. I confini, tuttavia, non vanno intesi come dei muri di recinzione, ma soltanto come confini di senso, che consentono di trattare le informazioni secondo le regole interne al sistema. I sistemi, pertanto, sono autonomi, ma non autarchici: attraverso l'autoreferenza il sistema genera i propri confini e mantiene la propria autonomia. Da ciò consegue che nella società moderna, di tutto ciò che accade si può dire se sta all'interno o all'esterno di un sistema, cioè di uno specifico orizzonte di senso. E' per questo che, infatti, la differenziazione del sistema sociale in sistemi parziali differenziati per funzione, non è altro che una differenziazione in specifici ambiti di senso: politico, giuridico, economico, religioso, scientifico, etc. La differenziazione funzionale si afferma, però, solo con la modernità.

Nel corso della evoluzione sociale, Luhmann individua altre tre forme della differenziazione, cioè altre forme in cui il sistema sociale riapplica a se stesso la distinzione sistema/ambiente. In questo modo, i sistemi sociali ottengono una differenziazione interna, cioè la differenziazione di diversi sottosistemi, ognuno ambiente per l'altro. Le altre tre forme della differenziazione sono: segmentaria

\section{Ivi, p. 225}

N. Luhmann, Illuminismo sociologico, cit., p. 75

R. De Giorgi, II mondo come sistema complesso, cit., pp. 63-64

N. Luhmann, Illuminismo sociologico, cit., p. 50

N. Luhmann, Sistemi sociali. Fondamenti di una teoria generale, cit., p. 632

N. Luhmann, Illuminismo sociologico, cit., p. 87 
(tipica delle società primitive e caratterizzata dall'uguaglianza dei sistemi parziali: famiglie, villaggi, tribù); centro/periferia (affermatasi nel periodo dei grandi imperi e caratterizzata da una comunicazione diffusa in modo diseguale sul territorio, a partire dalla città civilizzata alla campagna non civilizzata); infine, la differenziazione stratificata (dominante durante il medioevo e la prima modernità e caratterizzata dalla disuguaglianza di status dei sistemi parziali: nobiltà e popolo). Nella società moderna, tuttavia, le altra forme non scompaiono del tutto e infatti è possibile osservare zone centrali e zone periferiche della comunicazione economica, così come stratificazioni sociali e politiche all'interno dei confini territoriali: le comunicazioni giuridiche e quelle politiche, infatti, sono ancora segmentate in una moltitudine di Stati nazionali. Pertanto, quando si sostiene che la forma della differenziazione della società moderna è funzionale, si intende che i sistemi sono primariamente orientati all'assolvimento di una specifica funzione sociale. I sistemi si specificano funzionalmente e così si rendono autonomi sia sul piano strutturale (autorganizzazione) che su quello operativo (autopoiesi): ogni sistema è operativamente chiuso e quindi in grado di «specificare sotto quali aspetti [...] reagisce alle condizioni del suo ambiente, mentre da tutti gli altri aspetti si può permettere indifferenza grazie alla sua autopoiesi» ${ }^{51}$. La differenziazione funzionale della società moderna è semplicemente «la forma nella quale una elevata complessità sociale diventa organizzabile» ${ }^{52}$.

E' importante sottolineare che il passaggio da una forma all'altra di differenziazione sociale non avviene in modo programmato: l'evoluzione è una improbabilità che si rende probabile da sé. L'approccio evoluzionistico della teoria dei sistemi, infatti, «non ipotizza alcuno sviluppo necessario in senso storico o causale, ma opera con il concetto delle soluzioni vantaggiose dei problemi che, una volta stabilizzate, alleviano e facilitano l'esistenza umana al punto di essere difficilmente reversibili» ${ }^{53}$. II valore della storia consiste, pertanto, nella selettività già operata, nel fatto che nel presente non è possibile rinunciare ai sedimenti di senso del passato. Ciò comporta che «Tutto può essere modificato, ma non tutto in una volta ${ }^{54}$. Pertanto, l'impossibilità di programmare il futuro indica il ridimensionamento della politica nei confronti delle sue aspettative di intervento e di risoluzione dei problemi, poiché non è possibile più osservare gli eventi in base a relazioni causali punto per punto, ma in base ad una relazione complessa tra interdipendenze: in una società complessa, «la società produce trasformazioni senza poter controllare come tali trasformazioni si ripercuotano su di essa» ${ }^{55}$ e, quindi, la politica cessa di avere una responsabilità globale nei confronti del benessere della società. L'evoluzione si rende probabile da sè quando nuovi problemi creano un'emergenza di ordine che si stabilizza in una struttura non calcolata. Luhmann rinuncia così alla nozione causa/effetto e a quella mezzi/scopo, perché, a suo avviso, non è possibile attribuire ad una causa un singolo effetto e non è possibile attribuire ad un effetto una singola causa: come conseguenza, non si può costruire il futuro, pensare di adottare degli specifici mezzi per raggiungere uno scopo prefissato. Non ha senso, perciò, neanche «trattare le

N. Luhmann - R. De Giorgi, Teoria della società, cit., p. 22

N. Luhmann, Sociologia del diritto, cit. , p. 170

N. Luhmann Illuminismo sociologico, cit., p. 98

Ivi, p. 99

N. Luhmann, Teoria politica nello Stato del benessere, cit., p. 165 
trasformazioni sociali come progresso (in questo caso ci sono punti di riferimento esterni, costruiti dall'osservatore) considerarle cioè come sviluppo (intendendo unilinearità) ${ }^{56}$ : la società evolve come evolve, senza possibilità di programmarne il futuro.

La società moderna, pertanto, non è conseguenza di una programmazione, ma della crescente complessità della società, che rende avvertibile l'esigenza di nuove strutture selettive. I sistemi parziali, tuttavia, da una parte contrastano la contingenza del mondo, ma dall'altra «moltiplicano nella società le possibilità di costruire società» $^{57}$ : quindi, paradossalmente, la struttura di un sistema, nello stesso momento in cui riduce la complessità, la incrementa.

Le molteplici possibilità di costruire società sono possibili grazie alla comunicazione. Per questo motivo è rilevante accennare alla concezione innovativa che Luhmann ha della comunicazione. Egli si allontana dalla visione tradizionale soprattutto perché non concepisce la comunicazione come trasmissione di contenuti da un emissario ad un ricevente: il senso di una comunicazione, a suo avviso, non si trasmette, ma si produce. II concetto di senso, secondo la sua prospettiva, non andrebbe utilizzato come sinonimo di significato, ma indica ciò che si produce nella comunicazione sociale. Essa, pertanto, non trasferisce significati, ma è una operazione che produce senso e lo produce sempre e comunque, perché essa accade sempre. Se non ci fosse comunicazione, non ci sarebbero i sistemi sociali, la cui riproduzione autopoietica è garantita proprio dalle singole comunicazioni. Esse sono date dalla sintesi di tre elementi: emissione o atto del comunicare; informazione; comprensione. Quest'ultima, distinguendo tra emissione e informazione, fonda la comunicazione. La comprensione, però, non ha nulla a che vedere con l'intenzione di chi comunica che, per questo, non può essere considerata un elemento della comunicazione. L'idea diffusa secondo cui ci sarebbe qualcuno che ha qualcosa da comunicare e lo trasferisce a qualcun altro che comprende il senso, non è perciò più attuale. Secondo Luhmann, non si può trasferire senso da un sistema chiuso, come quello psichico, ad un altro sistema chiuso. Un sistema psichico si può osservare soltanto attraverso la comunicazione, proprio perché è chiuso, impenetrabile anche a se stesso: esso, quindi, può diventare solo tema di comunicazione. II comprendere, perciò, non è inteso come atto del recepire, ma del costruire. Quindi, ciò che si chiama senso si costruisce attraverso l'operazione della comunicazione, ma perché si possa osservare come senso è necessario che si attivi una nuova comunicazione. La stessa informazione, d'altra parte, non è un contenuto che si trasmette, recepisce e acquisisce, ma una distinzione che viene prodotta. L'atto del comunicare, pertanto, è qualsiasi atto che viene osservato e costruito come atto del comunicare, indipendentemente dall'intenzione dell'emissario. Ogni atto del comunicare è rumore, in virtù del quale si produce una differenza all'interno del sistema e il risultato è imprevedibile, nonché differente da sistema (psichico) a sistema (psichico).

Precisate queste premesse, Luhmann descrive la comunicazione come un evento improbabile, anche se accade sempre. A suo avviso, sono tre i livelli di improbabilità. II primo riguarda proprio l'improbabilità della comprensione. II 
secondo, la possibilità che l'emissione raggiunga l'interlocutore: tale improbabilità muta al variare della differenziazione sociale, perché come è evidente, in società primitive, l'interazione fra presenti facilita la probabilità del raggiungimento; la società moderna, invece, diventa tale grazie all'invenzione della stampa che per prima consente, - in modo diffuso -, di separare sul piano spaziale e temporale l'atto del comunicare e l'atto del comprendere. II terzo livello concerne l'improbabilità che una comunicazione venga accettata. Ciò che si chiede Luhmann è come mai sia possibile che una comunicazione improbabile venga poi resa probabile. A suo avviso, questo è reso possibile attraverso l'utilizzo dei media della comunicazione: il linguaggio, che rende probabile la comprensione; i mezzi di diffusione (stampa, radio, tv, internet, etc.) che rendono probabile il raggiungimento degli interlocutori; i mezzi di comunicazione simbolicamente generalizzati che, infine, rendono probabile l'accettazione.

Quelli che generalmente vengono chiamati mass media sono quindi ridefiniti da Luhmann media di diffusione della comunicazione ${ }^{58}$, onde evitare di confonderli con quelli che egli definisce media simbolicamente generalizzati, un'espressione, questa, che Luhmann mutua da Parsons. II sociologo statunitense utilizza il termine medium of interchange, ma lo scambio di cui si parla è uno scambio di prestazioni e di comunicazioni. Parsons, quindi, è il primo ad adottare il concetto di medium (implicitamente della comunicazione) in una teoria che non si occupi direttamente della comunicazione "mass mediatica", ma dei sistemi sociali. Fino ad allora, infatti, il termine era utilizzato all'interno di una branca specifica della sociologia: la sociologia della comunicazione. Essa aveva il compito di interpretare i cambiamenti che i cosiddetti mass media (stampa, radio, televisione) stavano producendo nella realtà sociale. Tuttavia, ad inizio anni sessanta, un altro studioso statunitense, Marshall McLuhan, interessato ad analizzare direttamente la comunicazione e i mass media, utilizza il termine medium in un senso più ampio:

«Tutto si riassume in un detto popolare che deriva a sua volta da una frase di Robert Browning: "La portata dell'uomo deve andare oltre la sua presa, altrimenti a che serve la metafora?". Tutti i media sono metafore attive in quanto hanno il potere di tradurre l'esperienza in forme nuove» 59 .

I media sono metafore. Attive, perché hanno il potere di formare la realtà, influendo profondamente sui rapporti umani. I media sono estensioni del nostro corpo che impongono «nuovi rapporti o nuovi equilibri tra gli altri organi e le altre estensioni del nostro corpo» ${ }^{60}$. Per questo, McLuhan estende la referenza semantica del concetto di medium e, prima di analizzare gli strumenti classici della comunicazione, analizza ogni tecnologia umana come un tentativo di andare oltre la presa semplice sul mondo: un tentativo, però, che cambia anche il nostro modo di esperire il mondo. Così, la ruota o la ferrovia, sono estensioni

58 Per un approfondimento della concezione luhmanniana dei mass media: Cfr. N. Luhmann, La realtà dei mass media, Franco Angeli, Milano 2002

59 M. McLuhan, Gli strumenti del comunicare, II Saggiatore, Milano 2008, p. 71. II titolo originale del libro, Understanding media (Comprendere $i$ media) evidenzia l'intenzione di McLuhan di ampliare la referenza semantica del concetto di media; il titolo italiano, invece, è completamente fuorviante perché riduce l'analisi di McLuhan ad una semplice riflessione sui mass media intesi in senso classico, come strumenti della comunicazione.

$60 \quad$ Ivi, p. 61 
dei piedi che modificano non solo la nostra possibilità di movimento, ma il nostro modo di pensare e di agire. «La ferrovia non ha introdotto nella società né il movimento, né il trasporto, né la ruota, né la strada, ma ha accelerato e allargato le proporzioni di funzioni umane già esistenti creando città di tipo totalmente nuovo e nuove forme di lavoro e di svago» ${ }^{61}$. L'idea innovativa di McLuhan è condensata, però, nella celebre frase II medium è il messaggio. Portando l'esempio della luce elettrica come di un medium senza messaggio, intende dimostrare che «il "contenuto" di un medium è sempre un altro medium ${ }^{62}$. I suoi effetti, quindi, non si verificano «al livello delle opinioni o dei concetti, ma alterano costantemente, e senza incontrare resistenza, le reazioni sensoriali o le forme di percezione» ${ }^{63}$. E' insensato, quindi, chiedersi se l'utilizzo "improprio" e monopolistico di un medium della comunicazione possa avere effetti di manipolazione sulle masse, perché non è l'utilizzo, più o meno buono di un medium, a manipolare: è il medium stesso che trasforma il vivere sociale e il modo in cui i sistemi psichici costruiscono e comunicano il mondo. A suo avviso, quindi, «il medium è il messaggio, perché è il medium che controlla e plasma le proporzioni e la forma dell'associazione e dell'azione umana. I contenuti, invece, cioè le utilizzazioni, di questi media possono essere diversi, ma non hanno alcuna influenza sulle forme dell'associazione umana» ${ }^{64}$. Secondo McLuhan, pertanto, non è necessario analizzare i contenuti che un medium veicola, o il suo utilizzo, ma il modo in cui un medium determina $i$ caratteri strutturali di una comunicazione e, conseguentemente, la struttura di una società.

Prima ancora di McLuhan, però, Parsons elabora un concetto di medium non strettamente connesso alla comunicazione (intesa in modo classico come uno specifico ambito di analisi). Per Parsons il medium non è specificatamente un medium di comunicazione, ma un medium di scambio. Nel testo Economia e società, Parsons concepisce il denaro come mezzo simbolico di scambio: è tramite esso che - nel suo celebre schema AGIL - si regolano i rapporti tra il sottosistema economico (A-daptation) e gli altri tre sottosistemi: il sottosistema politico (G-oal attainment), il sottosistema della comunità societaria (Integration) e il sottosistema educativo (L-atency). Analizzando gli interscambi tra i sottosistemi, Parsons teorizza la presenza di uno specifico medium di scambio in ogni sottosistema: il medium del potere nel sottosistema politico, il medium dell'influenza nella comunità societaria, e il medium degli impegni (o lealtà) nel sottosistema educativo. A suo avviso, è tramite uno scambio di prestazioni (e di comunicazioni) che i sistemi e i sottosistemi interagiscono tra loro.

Anche se la prospettiva di Parsons è completamente differente da quella di McLuhan, la sua idea che il medium sia simbolicamente generalizzato non è così distante dall'idea del medium come metafora. Infatti, con il termine simbolico, Parsons si riferisce alle chances che i media forniscono alla comunicazione perché questa possa essere accettata; con generalizzazione, invece, intende riferirsi alla validità dei media al di là di una specifica situazione. La funzione è la stessa svolta dal medium inteso come metafora attiva, perché 
sia il medium-simbolo che il medium-metafora hanno il potere di tradurre l'esperienza in forme nuove, cioè non vincolate ad una specifica situazione. Ogni metafora, d'altra parte, semplifica la complessità del mondo, tramite la sua riduzione ad una nuova immagine. Ogni metafora, quindi, ha una funzione selettiva.

Anche per questo motivo, nonostante Luhmann adotti da Parsons il concetto di medium simbolicamente generalizzato, la concezione luhmanniana dei media è - a nostro avviso -, paradossalmente più vicina alla concezione "strutturalista" di McLuhan che a quella di Parsons. Luhmann, infatti, accetta il senso dei termini così come era in Parsons, ma la sua teoria dei media della comunicazione «non si collega alla teoria parsonsiana dei media dell'interazione (o media of interchange), la quale resta vincolata alla architettura teorica dello schema AGIL ${ }^{65}$. I media di scambio simbolicamente generalizzati, concepiti da Parsons, diventano i media della comunicazione simbolicamente generalizzati: il denaro nel sistema economico, il potere nel sistema politico, la verità nel sistema scientifico, l'amore nel sistema familiare. La teoria dei media della comunicazione di Luhmann, d'altra parte, è molto più articolata rispetto a quella di Parsons, tant'è che la sua teoria della società è anche, o soprattutto, una teoria della comunicazione. Mentre Parsons utilizza la nozione di codice come equivalente del concetto di autorità, Luhmann concepisce il codice in relazione ai media della comunicazione. Egli, infatti, specifica che i media trasmettono prestazioni selettive tramite un codice di simboli generalizzati. Così come il codice del linguaggio descrive il mondo tramite distinzioni binarie che permettono di correlare ad ogni enunciazione positiva una corrispondente enunciazione negativa, allo stesso modo i media della comunicazione sono dei codici binari che orientano le comunicazioni di ogni sistema. Così il sistema della scienza si differenzia all'interno della società grazie all'orientamento al codice vero/non-vero, il sistema giuridico al codice lecito/illecito (o diritto/non-diritto), il sistema economico al codice avere/nonavere e così via. I codici non sono soltanto simbolici, ma anche diabolici, perché producono una differenza tra due valori: a differenza del medium del linguaggio, però, i codici dei media simbolicamente generalizzati sono anche preferenziali perché in essi uno dei valori è preferito socialmente in quanto positivo. Le preferenze consentono l'autocollocamento del codice in uno dei suoi valori, in modo tale da rappresentare l'unità del codice solo con una delle due parti. «L'autocollocamento del codice usa e rafforza per questo l'asimmetria del codice, la differenza tra valore positivo e valore negativo» ${ }^{66}$. II valore negativo (falso, torto, assenza di denaro, mancanza di amore) non viene però eliminato e quindi consente sempre la riflessione, poiché sottolinea la contingenza di quello positivo. Gli schematismi binari, perciò, non separano gli opposti, ma li collegano, in quanto tramite una negazione è possibile passare da una situazione al suo contrario. La codificazione binaria è quindi «una tecnica di integrazione paradossale» ${ }^{67}$ che, ad esempio, evidenzia la stretta connessione tra verità e falsità, cioè il fatto che la verità è un valore contingente che, in seguito, può diventare falsità. Con il termine tecnicizzazione, Luhmann indica «la facilitazione del passaggio dal valore al valore opposto» ${ }^{68}$. Grazie alla

Ivi, p. 144

N. Luhmann, Potere e complessità sociale, II Saggiatore, Milano 1979, p. 48

N. Luhmann - R. De Giorgi, Teoria della società, cit., p. 141 
codificazione secondaria è possibile ottenere un elevato grado di tecnicizzazione. Tale ulteriore codificazione consiste nella possibilità di duplicare il valore positivo. Ad esempio, il potere può raggiungere una codificazione secondaria tramite il diritto e così «si può usare il potere in modo conforme al diritto e in modo che violi il diritto» ${ }^{69}$.

La funzione principale dei codici si evidenzia, però, in relazione al problema della complessità. Luhmann, infatti, sostiene che tutti i media, e i loro codici, svolgono la funzione di strutture, in quanto rafforzano la selettività ${ }^{70}$ : i codici sono codici «di significati fissati come invarianti sulla base della elisione di altre possibilità ${ }^{71}$. Il codice del linguaggio è l'esempio più eclatante di struttura (selettiva), perché solo rispettando il suo codice, cioè evitando di riproblematizzare la struttura del linguaggio, è possibile comunicare: la comunicazione diventerebbe impossibile se in ogni interazione si mettessero in dubbio i significati delle parole e la struttura degli enunciati.

Strutture, media e codici, perciò, sono pre-selezioni che delimitano l'ambito delle possibilità di scelta, dentro un orizzonte di senso: essi scelgono ciò che è possibile scegliere e riducono quindi la complessità. Tuttavia, poiché il mondo è contingente, le possibilità pre-selezionate sono soltanto delle possibilità, perché altre possibilità sono state escluse, ma non eliminate: esse sono state solo rese latenti. La contingenza, pertanto, è rischio di delusione, perché spesso si realizzano solo eventi differenti da quelli attesi. Strutture e media, quindi, liberano «il singolo da buona parte dell'onere di un esame autonomo delle alternative ${ }^{72}$, perché le alternative vengono messe in ombra e quindi la loro stessa operazione di selettività resta latente. Esse, in definitiva,

«"ingannano" sulla vera complessità del mondo e [...] trasformano in questo modo il permanente sovraccarico provocato dalla complessità, nel problema di delusioni eventuali contro le quali è possibile intraprendere qualcosa. Dal punto di vista del sistema psichico si può quindi dire che le strutture regolano la pauras ${ }^{73}$.

Sulla base del concetto espresso nella precedente affermazione, Febbrajo ha sostenuto che la teoria dei sistemi di Luhmann adotti, paradossalmente, una ipotesi antropologica, perché attribuendo tanta rilevanza al concetto di complessità, finisce per utilizzare la paura e il conseguente bisogno di sicurezza come schemi interpretativi del comportamento umano ${ }^{74}$. Grazie all'influsso di Arnold Gehlen e di Helmut Schelsky, - continua Febbrajo -, «Luhmann sviluppa un concetto di struttura visto come quel meccanismo che serve a selezionare un ristretto campo di alternative di comportamento fra tutte quelle possibili allo scopo di consentire delle aspettative» ${ }^{75}$. Pertanto, anche a prescindere dalla paura o dal bisogno di sicurezza, le strutture diventano una necessità esistenziale perché fra tutte le alternative possibili, selezionano un campo di alternative di comportamento probabili: così è nel sistema della politica o in

\footnotetext{
IVi, p. 142

$70 \quad$ Cfr. N. Luhmann, Illuminismo sociologico, cit., pp. 149-150

$71 \quad$ R. De Giorgi, Scienza del diritto e legittimazione, cit., p. 227

72 N. Luhmann, Sociologia del diritto, cit., p. 50

$73 \quad$ IVi, p. 52

74 A. Febbrajo, Prefazione, in N. Luhmann, Sociologia del diritto, cit., pp. XIII-XIV

75 A. Febbrajo, Introduzione all'edizione italiana, in N. Luhmann, Sistemi sociali. Fondamenti di una teoria generale, cit., p. 17
} 
quello della famiglia o in quello dell'economia e così via. Scegliendo cosa è possibile scegliere, restringono il campo delle possibilità e rendono possibile la comunicazione, cioè coordinare il comportamento umano: rendono possibile non solo delle aspettative nei confronti di eventi, ma anche delle aspettative di aspettative, cioè aspettarsi le aspettative che altri hanno su di noi e di conseguenza agire in modo differente in base alla costruzione di tali aspettative di aspettative. E' per questo che Luhmann sostiene che «la sicurezza delle aspettative di aspettative [...] è il fondamento indispensabile di ogni interazione ed è assai più significativa della sicurezza dell'adempimento delle aspettative ${ }^{76}$. Per la probabilità della comunicazione, è molto più importante che si verifichi il meccanismo di aspettare delle aspettative, piuttosto che le aspettative effettivamente si realizzino. Eppure, anche le delusioni non possono verificarsi sempre, altrimenti provocherebbero la destabilizzazione dei sistemi sociali o la crisi di quelli psichici. Per questo motivo, ogni sistema dispone di due differenti strategie per reagire alle delusioni: «o mutare le aspettative deluse adattandole alla deludente realtà o tenerle ferme continuandole a vivere a dispetto della deludente realtà. $A$ seconda dell'atteggiamento che è dominante può parlarsi di aspettative cognitive o di aspettative normative ${ }^{77}$. In particolare, Luhmann specifica che per ottenere l'armonizzazione del comportamento sociale di più persone, cioè per sapere o prevedere in anticipo i tipi di comportamento possibili, è necessario che si creino delle aspettative valide non solo in situazioni specifiche. Per questo egli adotta il concetto parsonsiano di generalizzazione: «Attraverso la generalizzazione delle aspettative di comportamento viene facilitata la concreta sintonizzazione del comportamento sociale di più persone in quanto già è prefissato in maniera tipica ciò che ci si può aspettare, e quale comportamento farebbe saltare i confini del sistema»s ${ }^{78}$. Generalizzazione, pertanto, è indifferenza nei confronti delle differenze e quindi riduzione di complessità. Proprio il fatto che la generalizzazione (o stabilizzazione) avvenga al livello delle aspettative e non delle azioni, consente di trascendere una situazione specifica e di renderla valida anche nel futuro. Le aspettative normative, pertanto, sono quelle aspettative che vengono generalizzate nella dimensione temporale, poiché mediante normazione -, vengono mantenute stabili nel tempo in maniera controfattuale, cioè anche in presenza di una delusione o di un comportamento deviante. La generalizzazione nella dimensione materiale avviene attraverso l'identificazione del senso, senza tener conto della diversità delle aspettative. La generalizzazione sociale avviene, invece, tramite l'istituzionalizzazione: questo tipo di stabilizzazione poggia sulla aspettativa del consenso di terzi e quindi dà per scontata l'approvazione di una aspettativa, indipendentemente dal fatto che dei singoli possano non approvare: essa è una presupposizione di consenso.

In sintesi, tramite normazione, identificazione (del senso) e istituzionalizzazione, è possibile generalizzare temporalmente, materialmente e socialmente le aspettative, cioè ottenere una loro immunizzazione simbolica nei confronti di altre possibilità: la normazione assorbe le delusioni tramite sanzione; l'identificazione tramite programmi; l'istituzionalizzazione tramite procedimento. Pertanto, le aspettative vengono rese norme, tramite il meccanismo riduttivo

\footnotetext{
76 N. Luhmann, Sociologia del diritto, cit., pp. 48-49

$77 \quad$ Ivi, p. 53

78 R. De Giorgi, Scienza del diritto e legittimazione sociale, cit., p. 229
} 
delle sanzioni, perché siano stabili nel tempo contro le delusioni; vengono istituzionalizzate, tramite procedimenti (giuridici legittimanti), perché possa essere presupposto il consenso generale; infine, vengono fornite di senso perché ci si possa identificare. ${ }^{79}$ Tali meccanismi di generalizzazione, - che agiscono su durata, contenuto e consenso delle aspettative -, non operano, tuttavia, sempre in modo coordinato, ma «possono ostacolarsi e disturbarsi a vicenda. Tali incongruenze costituiscono un problema strutturale di ogni società, ed è in vista di questo problema che il diritto ha la sua funzione sociale ${ }^{80}$. In sostanza, affinché un'aspettativa possa diventare una norma giuridica è necessario che essa venga generalizzata non solo nella dimensione temporale, ma anche nelle altre due dimensioni: «A questo punto possiamo finalmente definire il diritto come la struttura di un sistema sociale che riposa sulla congruente generalizzazione di aspettative normative di comportamento» 81 .

Nella teoria di Luhmann, il diritto risulta quindi essere «un mezzo di integrazione dell'intera società e rappresenta, almeno nei confini territoriali dei sistemi politici, le aspettative di chiunque ${ }^{82}$. La funzione del diritto, pertanto, non consiste nella coercizione, quanto piuttosto nella facilitazione delle aspettative. Se proprio si volesse pensare al diritto in termini classici, interpretandolo in base alla funzione interdittivo-repressiva, allora bisognerebbe ritenere che la coercizione del diritto consiste nella selezione delle aspettative, come peraltro sostiene De Giorgi quando afferma che la razionalità del sistema giuridico consiste nella repressione: repressione di alternative, appunto. ${ }^{83}$ Riducendo la complessità, il diritto reprime e mantiene latente quegli spazi di realtà non funzionali al sistema. Tuttavia, - chiarisce De Giorgi -, il «negativo, il represso, l'emarginato, non deve apparire per quello che è, non deve apparire come realtà, perché sarebbe espressione appunto negativa della selezione [...]. II negativo deve apparire come possibilità positiva, come potenzialità di eventuale trasformazione. II represso così acquista una funzionalità positiva alla stabilizzazione del sistema» ${ }^{84}$. In questo modo, il represso acquista, paradossalmente, la funzione di distribuire speranze ai sistemi psichici, perché utilizza l'intrasparenza del futuro come medium per rendere accettabili le selezioni del presente. ${ }^{85}$

La repressione attuata dal diritto, tuttavia, avviene in ogni ambito sociale. Luhmann, infatti, non limita, come Parsons, «l'uso del concetto di struttura a

\footnotetext{
$79 \quad$ II problema della legittimità e del consenso viene risolto da Luhmann, nella dimensione sociale, tramite l'istituzionalizzazione: essa avviene - come si è accennato -, grazie ai procedimenti.

$80 \quad$ N. Luhmann, Sociologia del diritto, cit., p. 115

$81 \quad$ Ivi, p. 127.

$82 \quad$ IVi, p. 96

83 Cfr. R. De Giorgi, La razionalità come repressione e la teoria del diritto di N. Luhmann, in Id., Scienza del diritto e legittimazione sociale, cit.

Ivi, p. 258 Sul futuro come paradosso: Cfr. N. Luhmann, The Third Question: The Creative Use of
Paradoxes in Law and Legal History, in Journal of Law and Society, Vol. 15, n. 2, 1988; Sull'intrasparenza del futuro come meccanismo di creazione del consenso: Cfr. N. Luhmann, Metamorfosi dello Stato, in A. Cevolini (a cura di), Potere e modernità, Franco Angeli, Milano 2007, pp. 20-22
} 
modelli di comportamento stabilizzati normativamente» ${ }^{86}$ : non è solo il diritto a stabilizzare le aspettative, cioè a determinare socialmente il comportamento.

«La totalità delle premesse che nella società fungono da strutture non può essere ridotta unicamente a delle aspettative normative né, a maggior ragione, al solo diritto. [...] La società stessa non può essere compresa muovendo solo dalla sua costituzione giuridica. Il diritto è soltanto un momento strutturale tra gli altrì» $^{87}$.

La norma si definisce per «la disponibilità a comminare sanzioni nei casi di delusione» ${ }^{88}$, ma quando si parla di normazione non ci si riferisce esclusivamente alle norme (e alle sanzioni) del diritto, ma a tutte le aspettative normative presenti nei processi di interazione, tant'è che «in ogni società esistono più aspettative normative di quante possano essere istituzionalizzate» ${ }^{89}$. E' questo fabbisogno di aspettative normative che «produce le possibilità dell'aspettare normativo in vista delle quali il diritto può essere una struttura selettiva» ${ }^{90}$.

La normazione, quindi, è un fabbisogno sociale, sostenuto dal fatto che in ogni sistema sociale operano strutture, media e codici che scelgono cosa è possibile scegliere, che riducono la complessità del mondo rendendo possibile orientarsi, semplificando l'agire, fornendo delle strutture per praticare l'amore, per conoscere e dire la verità, per essere nel diritto. Ogni tentativo di fuga da una qualsiasi di queste strutture, ogni tentativo di rifiutare l'identità - cioè l'identificazione (per differenza) con il valore positivo del codice (verità, diritto, potere, amore) -, farebbe ricadere il fuggiasco nell'altra parte del codice e sarebbe immediatamente tematizzato come deviante e sottoposto a sanzione. Non è un caso, probabilmente, che Luhmann sottolinei che ogni codice non è solo simbolico, ma anche diabolico. L'etimo greco del verbo dia-ballo, infatti, indica il separare, ma anche il gettare o l'attraversare: il diavolo è colui che separa l'unità della distinzione, attraversa la demarcazione da una parte all'altra, scavalca la linea di confine della forma. Mentre i greci, però, vedevano nel daimon uno spirito non-negativo - per Socrate è uno spirito guida che lo assiste nelle decisioni -, nella cultura cristiana il diavolo diventa colui che getta calunnie contro Dio, perché Dio è l'unità della distinzione che ha reso determinato ciò che era indeterminato: «Come il peccato originale, così la forma produce un taglio nel mondo e la indeterminatezza che c'era prima diventa inaccessibile ${ }^{91}$. II cristianesimo, quindi, trasforma lo spirito-guida socratico nell'angelo sanzionato con la caduta dal paradiso perché ha osato separare l'unità e ha fatto vedere l'altra parte: l'illecito, la mancanza di amore, la mancanza di denaro e di verità, l'assenza di potere, ma, soprattutto, l'indeterminatezza, la mancanza di preselezioni, le infinite possibilità.

«Attraversare è un atto creativo» ${ }^{92}$, dice Luhmann. Tuttavia, quando nella società si cerca di compiere questo atto creativo (e diabolico), e si cerca quindi di separare la distinzione o di attraversarla da una parte all'altra, si incorre in

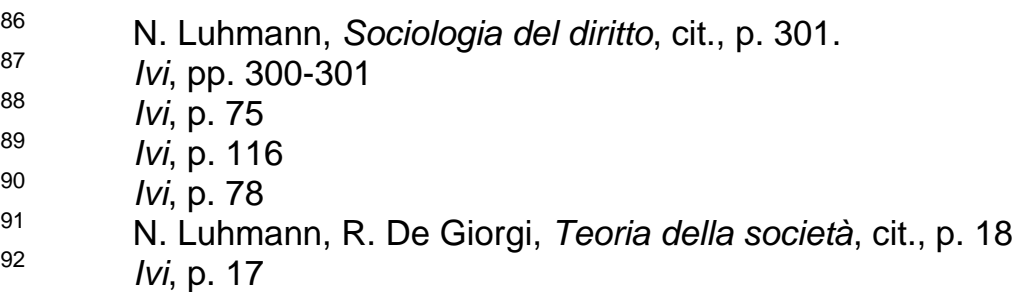


sanzione. Nel caso in cui il tentativo di attraversamento interessa il codice del sistema giuridico, la devianza viene costruita come illecito, il deviante come criminale e la sanzione come pena. Più interessante, invece, è verificare cosa accade quando il tentativo di attraversamento interessa le operazioni di qualsiasi altro sistema sociale: in questo modo, infatti, è possibile verificare che «il diritto è soltanto un momento strutturale tra gli altri». In un rapporto sentimentale, ad esempio, il tradimento delle aspettative del proprio partner viene facilmente interpretato come valore negativo del codice, cioè come mancanza di amore. D'altra parte, tradire deriva dal latino tràdere, composto dalla particella trans che indica oltre, al di là, e da dère, cioè dare, consegnare: tradire, quindi, può essere interpretato come un andare al di là del valore positivo del codice, un dare l'altra parte: il non-amore. Un andare oltre la struttura dell'amore che può provocare o la difficile ri-problematizzazione della struttura e quindi una sua evoluzione o, come spesso accade, la sanzione, cioè l'interruzione della relazione, il crollo della struttura e, contemporaneamente, di qualche sistema psichico. Pertanto, affinché la struttura resti stabile e i sistemi psichici possano avere una sufficiente dose di sicurezza contro la paura, i due partners si impegnano costantemente per costruire le aspettative dell'altro e per rispettare quella che si sedimenta come struttura dell'amore e che orienta nell'agire, dice cosa è sperimentabile e cosa no. E' molto più probabile, quindi, che i partners di una relazione si impegnino volutamente nel mantenimento della struttura così com'è, nell'accettare le sue preselezioni, nel contribuire alla sua istituzionalizzazione: chi volesse tentare di ri-problematizzare la struttura, infatti, non solo dovrebbe sopperire alla mancanza di tempo e alla scarsa capacità di attenzione (propria e dell'altro), ma «ha contro di sé il peso, difficilmente bilanciabile, di una pretesa ovvietà. [...] Egli attacca, quindi, autorappresentazioni altrui e diventa scomodo, se non pericoloso» ${ }^{93}$. Per questo motivo, è molto più probabile che si scelga di non deludere quelle che vengono costruite come aspettative dell'altro, mentre l'altro contemporaneamente opera allo stesso modo: e mentre entrambi costruiscono le aspettative dell'altro e si comportano in base a questa invenzione, può accadere, paradossalmente, che entrambi abbiano intimamente aspettative completamente opposte da quelle costruite dall'altro e quindi che entrambi abbiano aspettative coincidenti. Tuttavia, se questa coincidenza non viene mai comunicata, o viene comunicata e costruita dall'altro in modo differente, accade che le aspettative dei partners si ritrovano coincidenti nel punto esattamente opposto a quello inizialmente desiderato da ogni singolo sistema psichico: questo ipotetico punto (o rete di comunicazioni) in cui le aspettative coincidono e trovano un loro controllo e un loro ordine è il sistema (sociale). Quanto più ogni sistema psichico si rifiuta di affrontare la complessità o la paura, tanto più è probabile che ognuno si rinchiuda nelle preselezioni della struttura, come in una prigionia volontaria ${ }^{94}$, in cui ognuno crede di obbedire a se stesso o a un'altra parte di sé che può inventare come spirito-guida. E invece, ognuno sta obbedendo al sistema. The control's control is the system.

Ogni medium comunica la sua struttura. II medium è il messaggio. II messaggio non esiste, perché il messaggio è il sistema, la sua struttura. Amore, potere,

\footnotetext{
$93 \quad$ N. Luhmann, Sociologia del diritto, cit., p. 85

94 Il concetto di prigionia volontaria è utilizzato da Elias Canetti per spiegare l'obbedienza. Cfr. E. Canetti, Massa e potere, Adelphi, Milano 1973
} 
verità, diritto scelgono cosa è possibile scegliere e, con i loro codici diabolici e preferenziali, suggeriscono solo una parte della distinzione e occultano l'altra parte in un giardino dell'eden pagano. Un giardino che rimane possibile, ma anche altamente improbabile, quantomeno per i nostri corpi organici e per i nostri sistemi psichici che, inchiodati in questo presente, non avranno forse il tempo per sperimentare l'altra parte dei codici; né, soprattutto, avranno il tempo perché altre strutture del potere, dell'amore, della verità e del diritto, si rendano probabili da sé. A meno che, i nostri stessi sistemi psichici, con un atto creativo, non vogliano costruirsi come liberi e rischiare, però, di vedere un luogo e un volto del potere, e di essere tematizzati come folli o come indemoniati: rinchiusi in un ambiente molto simile ad una gabbia, in una prigionia affatto volontaria. $\mathrm{E}$ affatto metaforica. 